\title{
How explosive are cryptocurrency prices?
}

\author{
Marc Gronwald*
}

May 2020

\begin{abstract}
This paper revisits the issue of price explosiveness in cryptocurrency markets by analysing the prices of Bitcoin, XRP, Ether, and Litecoin not only expressed in US Dollars, but also the price of the latter three expressed in Bitcoin. There is strong evidence of explosive periods in cryptocurrency prices expressed in US Dollars, but also when $\mathrm{XRP}$ and Ether are expressed in Bitcoin. Whether or not these explosive periods can be interpreted as cryptocurrency bubbles requires a sufficient understanding of the fundamental value of cryptocurrencies. As this is currently insufficient, the term bubble should be used with more caution.
\end{abstract}

Keywords: Cryptocurrencies, Bubbles, Explosiveness, Fundamental value, Intrinsic value, Fiat money

JEL-Classification: C12, C22, E42, E52, G12

${ }^{*}$ University of Aberdeen Business School, CESifo and ifo Institute. Email: mgronwald@abdn.ac.uk. The author gratefully acknowledges useful comments by Beat Hintermann, Xin Jin, as well as participants of the 18th Journees LAGV - International Conference in Public Economics. Furthermore, the author is indebted to Sandrine Ngo for motivating me to study the economics of Bitcoins. 


\section{INTRODUCTION}

Motivated by frequent enormous price increases cryptocurrencies such as Bitcoin exhibit, a lively debate emerged whether or not there are cryptocurrency bubbles. Any empirical test for asset price bubbles generally consists of two steps. First, the empirical properties of the price series have to be evaluated. A very common procedure for this is the test for (temporary) explosiveness proposed by Phillips et al. (2015). Second, as a bubble is usually defined as deviation of an observed price from its fundamental value (Diba and Grossman, 1988), any statement about an asset price bubble requires having an understanding of what the fundamental value of the underlying asset is.

This paper makes two contributions to this discussion. The first is the application of Phillips et al.'s (2015) test not only using the prices of the leading cryptocurrencies Bitcoin, Ether, XRP, and Litecoin expressed, as conventional, in US Dollars, but also by analysing the prices of the latter three currencies expressed in terms of Bitcoin. ${ }^{1}$ Thus, this paper analyses whether or not a stable relationship exists between prices of different cryptocurrencies, or, in other words, if prices changes of the different currencies follow the same pattern and extent. It is directly related to a literature that deals with the relationship of prices of different currencies, epitomised by papers such as Bouri et al.'s (2019b) study on herding behavior in cryptocurrencies, Bouri et al.'s (2018) analysis of co-explosivity in the cryptocurrency market, and Bouri et al. (2019c) which deals with co-jumping behaviour

\footnotetext{
${ }^{1}$ Ether and XRP are the cryptocurrencies generated by the platforms Ethereum and Ripple, respectively.
} 
of cryptocurrency returns. The results obtained in this paper can be summarised as follows: First, evidence for temporary explosiveness is found in prices of all currencies under consideration expressed in US Dollars. This finding is largely in line with the existing literature. However, the price of both Ether and XRP is also found to be temporarily explosive even if expressed in terms of Bitcoin. This means that the price changes of these two cryptocurrencies are disproportionally larger than changes in Bitcoin prices in the respective periods. It is worth highlighting that this feature is only observed during earlier cryptocurrency episodes in the first half of 2016 and 2017, respectively, but not during the price peak period of Bitcoin exhibited around the turn of the year $2017 / 2018$. The price of Litecoin expressed in Bitcoin, in contrast, does not show this peculiar behaviour.

The second contribution this paper makes is a discussion of what an analysis of this type allows one to say regarding bubbles in cryptocurrencies. It has already been stated that an asset price bubble is conventionally defined as an asset price that diverges from its fundamental value (Diba and Grossman, 1988). Thus, the essential question in this context is the following: what is the fundamental value of a cryptocurrency? This question has been insufficiently addressed in the existing literature. For example, both Cheung et al. (2015) and Geuder et al. (2019) follow Phillips et al. (2015) and assume that evidence of price explosiveness implies that there is evidence of a bubble. However, Phillips et al.'s (2015) procedure is based on a theoretical stock price model which gives explicit consideration to determinants of the fundamental value of stock prices as well as empirical properties of those determinants. Explosiveness can only be interpreted as evidence of a bubble 
under certain conditions. A discussion is required whether nor not this also applies in the cryptocurrency case. What is more, Cheah and Fry (2015) estimate the fundamental value of Bitcoin from data rather than using any theoretically derived fundamental value as benchmark at all. In a nutshell, unless a better understanding of the fundamental value of cryptocurrencies has been achieved, the term bubble should be used with more caution.

The remainder of the paper is organised as follows: Section 2 presents the empirical analysis and Section 3 discusses what that analysis allows one to say about a cryptocurrency bubble. Section 4 concludes this paper by discussing the general messages that emerge from this paper.

\section{TESTING FOR EXPLOSIVEnESS}

Bitcoin emerged in 2009; followed by various other cryptocurrencies. Drastic increases in the value of many of these cryptocurrencies occur very frequently. Figure 1a presents the prices of Bitcoin, Ether, XRP, and Litecoin; all expressed in US Dollars. ${ }^{2}$ This phenomenon motivated many to empirically test for the existence of bubbles in cryptocurrency prices. ${ }^{3}$

Carefully inspecting Figure 1a yields that price hikes of these currencies seem to occur simultaneously. This is not surprising insofar as cryptocur-

\footnotetext{
${ }^{2}$ This data-set is used throughout this paper. The period of observation is $07 / 08 / 2015$ 25/11/2019; all data from www.coinmarketcap.com, a standard data source also used by e.g. Geuder et al. (2019) and Bouri et al. (2019b). The cryptocurrencies selected here are all within the Top 7 in terms of market capitalisation. Tether has not been included as it is a so-called stablecoin. In addition, coins such Bitcoin cash have been ignored as they are merely forks of Bitcoin.

${ }^{3}$ This literature on cryptocurrency bubbles is an offshoot of a recently emerged enormous empirical literature on Bitcoin and other cryptocurrencies; see Gronwald (2019) for one of the most recent contributions. That paper also provided a comprehensive overview of the literature.
} 
Figure 1: Cryptocurrency prices expressed in US Dollars and in Bitcoin
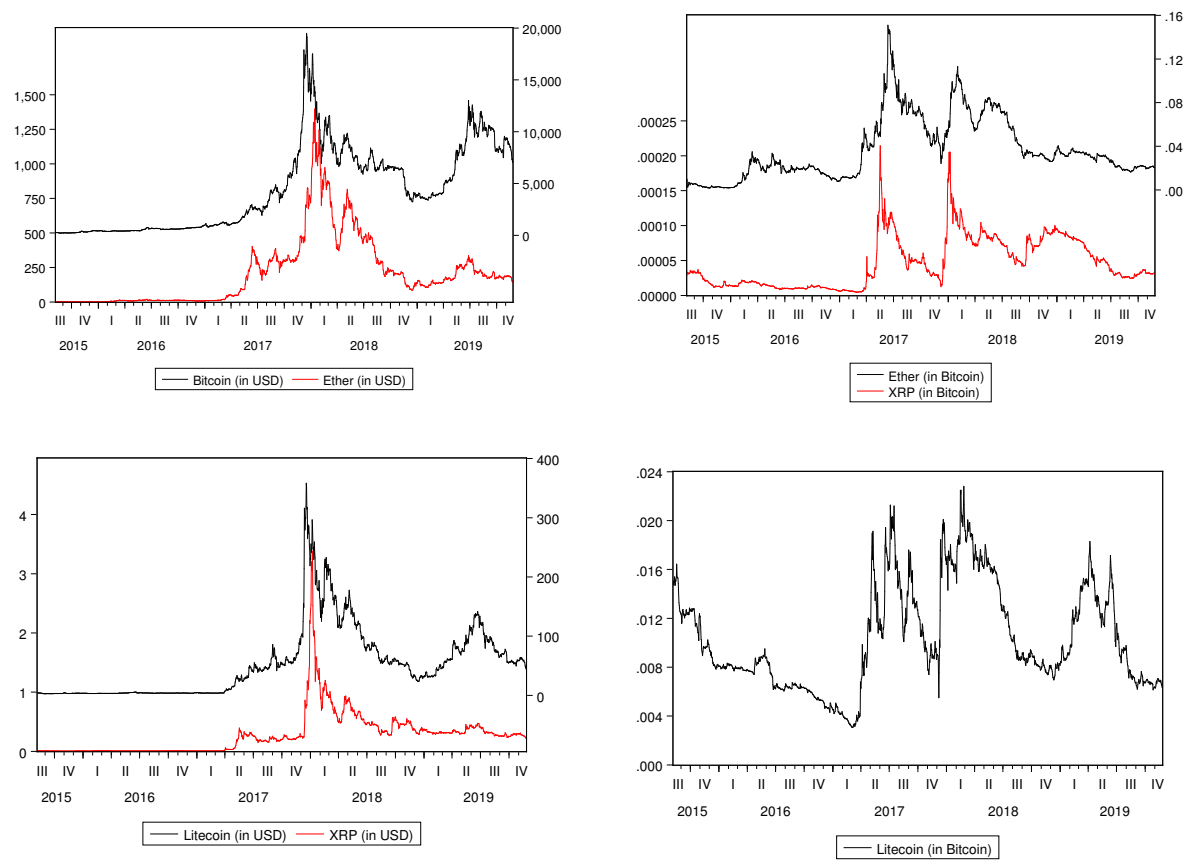

(a) Cryptocurrency prices in US Dollars

(b) Cryptocurrency prices in Bitcoin

rencies are largely considered speculative assets, and, thus, all price changes follow the same overall pattern. For this reason it would not be implausible to assume that there is a more or less stable relationship between these price series. However, Figure 1b vividly illustrates that this is not the case. Presented are the price of XRP, Ether, and Litecoin expressed in terms of Bitcoin. ${ }^{4}$ It is evident that these price series is far from stable. There are certainly rather horizontal movements in some periods such as 2016 and the second half of 2018, but also drastic changes in these series. These changes

\footnotetext{
${ }^{4}$ By expressing Ether, XRP, and Litecoin in terms of Bitcoin, it is assumed that the most polular and oldest cryptocurrencies, Bitcoin, is the leading cryptocurrency.
} 
occurred during similar periods as those the cryptocurrencies expressed in US Dollar exhibited drastic increases. To summarize, even though the pattern of the price changes is overall similar, there are considerable differences in their extent across cryptocurrencies. These price series are now analysed using Phillips et al.'s (2015) procedure. Hence, this paper's analysis of the relationship between cryptocurrency prices contributes to an offshoot of the cryptocurrency literature which so far has has focussed on issues such as volatility spillovers (Yi et al., 2018), co-explosivity (Bouri et al., 2018), co-jumping (Bouri et al., 2019c) as well as herding behaviour in cryptocurrencies (Bouri et al., 2019b).

This standard procedure consists of a forward recursive application of an augmented Dickey-Fuller unit root test. The null of a unit root is tested against the alternative of an explosive root. Thus, the following equation is estimated:

$$
x_{t}=\mu_{x}+\delta x_{t-1}+\sum_{j=1}^{J} \phi_{j} \Delta x_{t-j}+\epsilon_{x, t}, \quad \epsilon_{x, t} \sim \operatorname{NID}\left(0, \sigma_{x}^{2}\right) .
$$

The hypothesis $H_{0}: \delta=1$ is tested against the alternative $H_{1}: \delta>1 .^{5}$ Initially, a subset of the sample with $\tau_{0}=n r_{0}$ observations is used. In each subsequent regression, this subset is supplemented by successive observations, giving a sample of size $\tau=n r$ for $r_{0} \leq r \leq 1$. This procedure yields a sequence of $t$-statistics with corresponding p-values. These sequences are used to identify origination $\hat{r}_{e}$ and collapse dates $\hat{r}_{f}$ of explosive behavior in

\footnotetext{
${ }^{5}$ Note that this is a standard unit root test except for the formulation of the alternative hypothesis. Rather than testing the null of a unit root against a stationary alternative, the alternative in this case is explosive.
} 
Figure 2: Tests for explosiveness: Bitcoin, Ether, XRP, and Litecoin expressed in USD
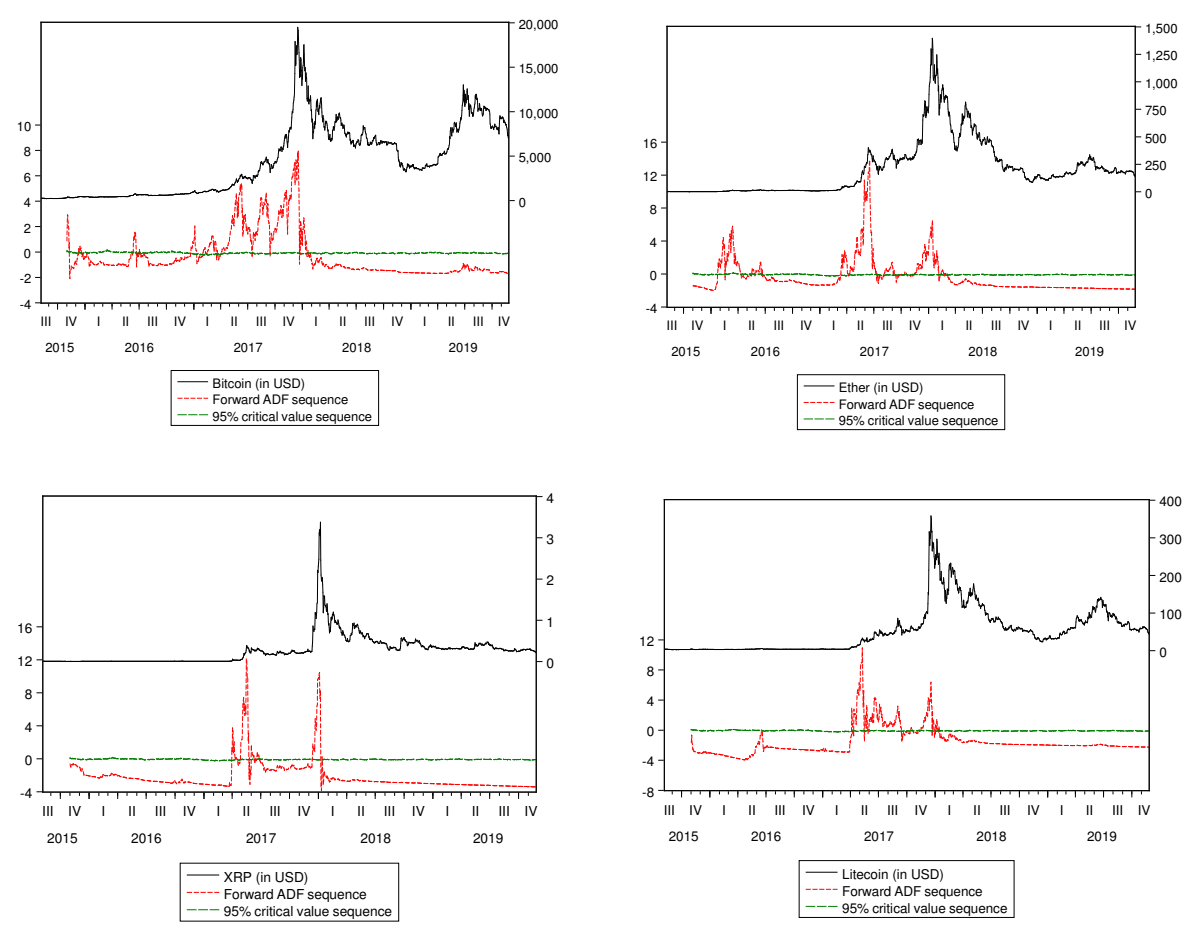

the data:

$$
\begin{aligned}
& \hat{r}_{e}=\inf _{s \geq r_{0}}\left\{s: \operatorname{ADF}_{s}>\operatorname{cv}_{\beta_{n}}^{\operatorname{adf}}(s)\right\} \\
& \hat{r}_{f}=\inf _{s \geq \hat{r}_{e}}\left\{s: \mathrm{ADF}_{s}<\operatorname{cv}_{\beta_{n}}^{\operatorname{adf}}(s)\right\}
\end{aligned}
$$

where $\operatorname{cv}_{\beta_{n}}^{\operatorname{adf}}(s)$ stands for the critical value. ${ }^{6}$

Figures 2 and Figure 3 present the results. Each panel displays the

\footnotetext{
${ }^{6}$ In the empirical application, the critical values are simulated usung the Monte Carlo technique; see Phillips et al. (2015).
} 
Figure 3: Tests for explosiveness: Ether, XRP, and Litecoin expressed in Bitcoin
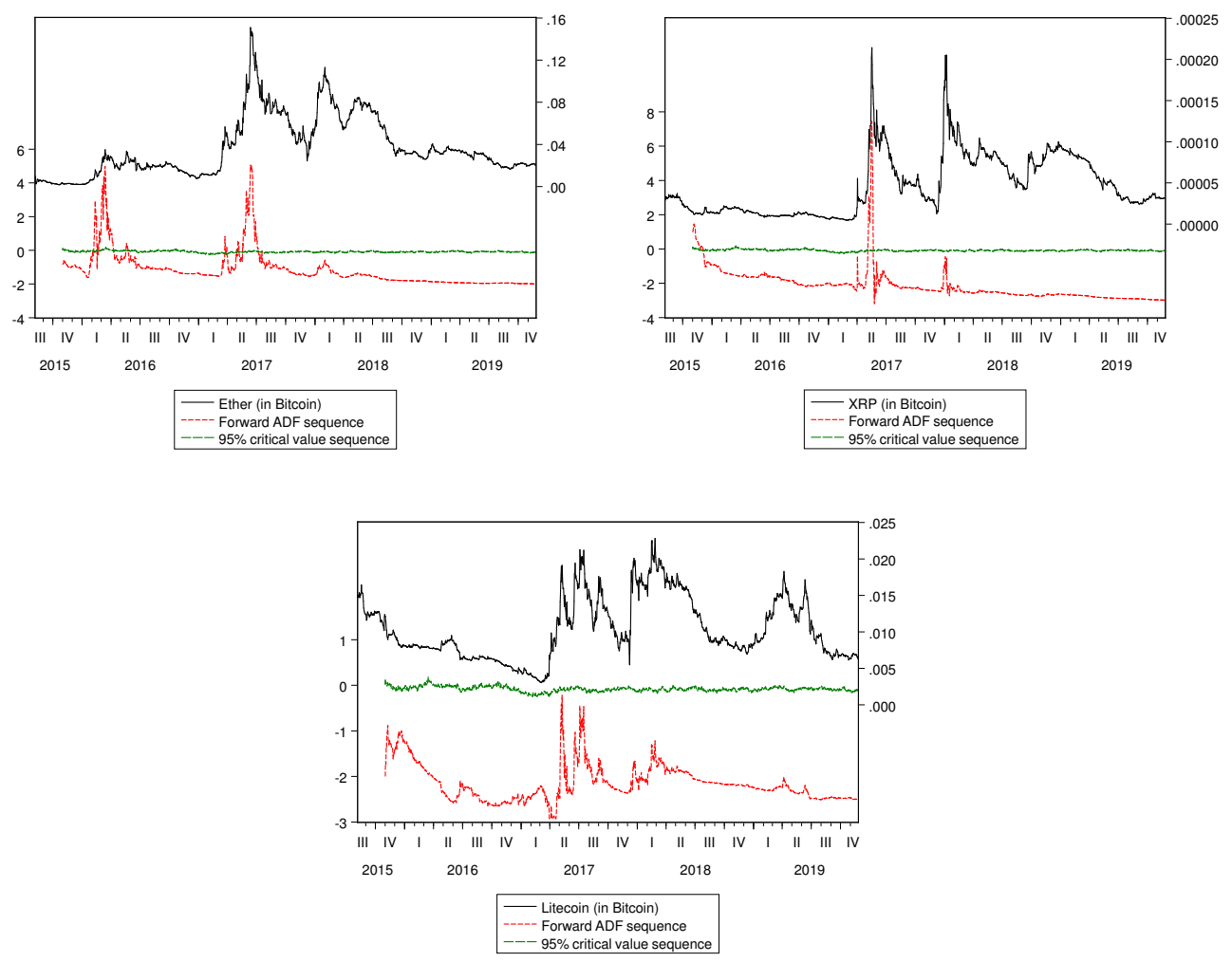

ADF sequence as well as the critical values for a cryptocurrency quoted in US Dollars (Figure 2) and in Bitcoin (Figure 3). It is evident that all cryptocurrency prices expressed in US Dollars exhibit temporary phases of explosiveness, in particular in 2017. This finding is generally consistent with the extant literature. What is more, evidence of temporary explosiveness is also found in the prices of XRP and Ether expressed in Bitcoin. However, these periods are found to be only in early stages of 2017, and, in addition, in early 2016, but not during the price peak period of Bitcoin at the turn 
of the year 2017/2018. The interpretation is the following: the prices of $\mathrm{XRP}$, Ether and Bitcoin are generally following the same pattern; however in certain periods the prices of XRP and Ether are explosive even if expressed in terms of Bitcoin. This reflects that in these periods the change in XRP as well as Ether prices are disproportionally larger than change in Bitcoin prices. It is worth highlighting that the price of Litecoin does not exhibit this idiosyncratic behaviour.

This particular aspect has not yet been addressed in the extant literature that deals with the relationship between prices of different cryptocurrencies. Yi et al. (2018), for instance, find increasing degrees of connectedness between different cryptocurrencies and, in addition, are able to identify the direction of volatility spillover. ${ }^{7}$ Bouri et al. (2019a) analyse causality linkages among volatility surprises of a number of leading cryptocurrencies and find that not only volatility surprises of the largest cryptocurrency, Bitcoin, matters but also those in smaller currencies. Noteworthy is, however, Bouri et al.'s (2019b) analysis of herding behaviour in cryptocurrencies. They find evidence of herding, but mainly in the period April 2016 - September 2017. The additonal analysis they conduct reveals that uncertainty is an important driver of this result. Herding describes the phenomenom that decisions to invest in certain cryptocurrencies is not only driven by the characteristics of one individual currency but by the attractiveness of the market as a whole. The extreme price movements of XRP and Ether identified in this paper could certainly reflect herding behavior, in particular as they are found to be in periods prior to those where the cryptocurrency prices peaked

\footnotetext{
${ }^{7}$ See Omane-Adjepong and Alagidede (2019) for a similar analysis.
} 
at the turn of the year. In those periods, data on trading volumes suggest that interest in these markets was generally not as high yet. Thus, spikes in demand for certain cryptocurrencies due to herding behaviour can very well explain the observed price patterns. ${ }^{8}$ Finally, Bouri et al. (2019c) investigate whether or not prices of different cryptocurrencies jump together; Bouri et al. (2018) is concerned with co-explosive behavior. In other words, both these papers deal with specific types of extreme price behavior which, however, occurs contemporaneously. This paper explicitly addresses a different issue: the focus is on whether or not a stable relationship is found, or, in other words, if price changes of different cryptocurrencies are of the same extent. In those periods prices of XRP and Ether are found to be explosive even if expressed in terms of Bitcoin, neither is the case.

\section{Discussion}

This section discusses to what extent the findings obtained in Section 2 allow one to draw conclusions regarding the existence of cryptocurrency bubbles. Centre stage in this discussion takes the notion that any analysis of an asset price bubble requires sufficient knowledge about the fundamental value of the respective asset. It has been asserted above that the existing literature on cryptocurrency bubbles insufficiently takes this into account. Both the early contribution by Cheung et al. (2015) and the more recent one by Geuder et al. (2019) follow Phillips et al. (2015) and assume that evidence of price explosiveness implies evidence of a bubble also in the context of cryptocurrencies. It should be noted, however, that Phillips et al. (2015) - and

\footnotetext{
${ }^{8}$ It falls outside the scope of this short paper to investigate this in more detail.
} 
also Diba and Grossman (1988), the seminal paper their analysis is based on deal with stock market bubbles. Both papers propose theoretical stock price models; in both papers the fundamental value of stock prices is determined by, first, future dividend payments and, second, some unobservable fundamental factors. What is more, both papers invest considerable effort into discussing the empirical properties, i.e. stationarity vs. non-stationarity, of these determinants. The conclusion that evidence of explosiveness can be interpreted as evidence of a bubble only holds under certain conditions. In a nuthell, the fundamental value of stock prices in papers such as Phillips et al. (2015) is not only based on economic theory, it is also measurable using financial market data. What is more, Gronwald's (2016) analysis of the crude oil market shows that also the fundamental value of an asset can change drastically. The literature on cryptocurrency bubbles pays insufficient attention to the question whether or not the fundamental value of cryptocurrencies can be modelled using Phillips et al.'s (2015) asset pricing model and, in addition, what the statistical properties of the determinants of the fundamental value of cryptocurrencies are. Finally, Cheah and Fry (2015) also find evidence of cryptocurrency bubbles. In addition, they employ an empirical procedure to estimate the fundamental value of Bitcoin and find that this value is equal to zero. This is highly problematic insofar as this fundamental value is derived from observed prices and not based on any economic theory. ${ }^{9}$

\footnotetext{
${ }^{9}$ It should also be noted that some papers are more careful in this regard. Corbet et al. (2018), for instance, undertake the attempt to explicitly take the fundamental value into account; their approach is based on blockchain position, hashrate, and liquidity. They construct three measures that are supposed to capture "key theoretical components of cryptocurrency pricing structures". Bouri et al. (2018), in addition, emphasize that, due
} 


\section{Conclusions}

It is an essential question whether or not asset prices appropriately reflect market fundamentals as, for instance, information provided by financial markets is used for making investment decisions. The existence of a bubble would, however, not only mean that asset price do not correctly reflect market fundamental; it has more far-reaching consequences. Concerns about the existence of bubbles potentially also influences the public's opinion on the role of financial markets in an economy. What is more, concerns about bubbles potentially sparks governmental regulators to intervene into the markets. For all these reasons it is essential that bubbles are correctly identified. Various examples can be found for public as well as academic debates sparked by rapidly increasing prices. A plethora of studies deals with stock market bubbles, epitomised by Phillips et al. (2015). The crude oil market also attracts attention, see e.g. Gronwald (2016); additional markets that come to mind include real estate markets and other commodity markets. Ever since the emergence of cryptocurrencies, these markets have also been scrutinised. In light of the fact that price movements on these markets are extraordinary, this is no surprise. There is overwhelming evidence that cryptocurrency prices are characterised by (temporary) explosiveness. This paper confirms these findings, but also finds that some cryptocurrency prices are explosive even if expressed in terms of Bitcoin and not, as conventionally, in US Dollars. In addition, this paper points out that whether or not the explosive periods can be interpreted as bubbles remains unclear

to the lack of clarity in this issue, an analysis of a cryptocurrency bubble is not possible; in consequence, they focus on co-explosivity. 
as the fundamental value of cryptocurrencies is insufficiently understood. The popular test for (temporary) explosiveness proposed by Phillips et al. (2015) has not only originally been applied in the area of stock markets, but is also based on theoretical stock price modelling and empirical properties of observable and unobservable market fundamentals. As the fundamental value of cryptocurrencies is insufficiently understood, it remains unclear if this procedure can easily be applied in the context of cryptocurrencies.

It is, however, not the case that nothing can be said about the fundamental value of cryptocurrencies from an economic perspective. Cryptocurrencies share with fiat money the feature that both have a positive value despite the fact that their respective intrinsic values are zero. This essentially reflects Ali et al.'s (2014) assertion that "digital currencies have meaning only to the extent that participants agree that they have meaning". Hence, a parallel can be drawn to the discussion about the fundamental value of money. Tirole's (1985) seminal paper, for instance, states that the market fundamental of money is equal to the present discounted value of transaction savings, and is, thus, based on theoretical economic considerations. Tirole (1985) summarises his discussion in the so-called fundamentalist view: if money is used for transactions, it must be a store of value, and, in that case, there is no bubble on money. Starr (1974) offers an alternative view by stating that money is needed to pay income tax and, thus, there cannot be a bubble on money. Tirole (1985) and also Stiglitz (1990) certainly also state that, if money is only held for speculative purposes and not for transactions, there is a bubble in money. ${ }^{10}$ Some of these arguments reappear in

\footnotetext{
${ }^{10}$ Tirole (1985) discussion of Flood and Garber (1980) shows that bubbles in money are
} 
the recent paper by Bolt and van Oordt (2019). They show that the value of cryptocurrencies is determined by three components two of which are the value of transactions in that currency and decisions by forward-looking investors (that affect supply of the currency). ${ }^{11}$ They, however, also state not much is known about the actual number of payments in virtual currency for goods and services. Worth highlighting is Bolt and van Oordt's (2019) "hypothetical Bitcoin exchange rate in the absence of speculation". The finding that this rate substantially deviates from the actual exchange rate should be seen as evidence of a Bitcoin bubble. It should also be noted, however, that this hypothetical exchange rate exhibits steep increases resembling those in the actual exchange rate. As Bolt and van Oordt (2019) themselves acknowledge, more research in this area is required; however this is certainly an interesting starting point. ${ }^{12}$ In a nutshell, it seems to be difficult to determine the fundamental value of cryptocurrencies. As long as this remains the case, the term cryptocurrency bubble should be used with caution.

\section{REFERENCES}

Ali, R., Barrdear, J., R.Clews, and Southgate, J. (2014). The economics of digital currencies. Bank of England Quarterly Bulletin, pages 1-11.

Bolt, W. and van Oordt, M. (2019). On the value of virtual currencies.

difficult to detect.

${ }^{11}$ The third is "the elements that jointly drive future consumer adoption and merchant acceptance of virtual currency".

${ }^{12}$ See Appendix A in Bolt and van Oordt (2019) for a critical discussion of this hypothetical exchange rate. 
Journal of Money, Credit, and Banking, DOI: 10.1111/jmcb.12619.

Bouri, E., B.Lucey, and Roubaud, D. (2019a). The volatility surprise of leading cryptocurrencies: Transitory and permanent linkages. Finance Research Letters, fortcoming.

Bouri, E., Gupta, R., and Roubaud, D. (2019b). Herding behavoir in cryptocurrencies. Finance Research Letters, 29:216-221.

Bouri, E., Roubaud, D., and Shahzad, S. (2019c). Do Bitcoin and other cryptocurrencies jump together. The Quarterly Review of Economics and Finance, forthcoming.

Bouri, E., Shahzad, S., and Roubaud, D. (2018). Co-explosivity in the cryptocurrency market. Finance Research Letters.

Cheah, E. and Fry, J. (2015). Speculative bubbles in Bitcoin markets? an empirical investigation into the fundamental value of bitcoin. Economics Letters, 130:3236.

Cheung, A., Roca, E., and Su, J.-J. (2015). Crypto-currency bubbles: An application of the Phillips-Shi-Yu (2013) methodology on Mt. Gox Bitcoin prices. Applied Economics, 47(23):2348-2358.

Corbet, S., Lucey, B., and Yarovaya, L. (2018). Datestamping the Bitcoin and Ethereum bubbles. Finance Research Letters, 26:81-88.

Diba, B. and Grossman, H. (1988). Explosive rational bubbles in stock prices? The American Economic Review, 78(3):520-530. 
Flood, R. and Garber, P. (1980). Market fundamentals versus price-level bubbles: The first tests. Journal of Political Economy, 88:745770.

Geuder, J., Kinateder, H., and Wagner, N. (2019). Cryptocurrencies as financial bubbles: The case of Bitcoin. Finance Research Letters, 31:179184.

Gronwald, M. (2016). Explosive oil prices. Energy Economics, 60:1-5.

Gronwald, M. (2019). Is Bitcoin a commodity? On price jumps, demand shocks and certainty of supply. Journal of International Money and Finance, https://doi.org/10.1016/j.jimonfin.2019.06.006.

Omane-Adjepong, M. and Alagidede, I. (2019). Multiresolution analysis and spillovers of major cryptocurrency markets. Research in International Business and Finance, 49:191-206.

Phillips, P., Shi, S., and Yu, J. (2015). Testing for multiple bubbles: historical episodes of exuberance and collapse in the S\&P500. International Economic Review, 56(4):1043-1077.

Starr, R. (1974). The price of money in a pure exchange monetary economy with taxation. Econometrica, 42:45-54.

Stiglitz, J. (1990). Symposium on bubbles. Journal of Economic Perspectives, $4(2): 13-18$.

Tirole, J. (1985). Asset bubbles and overlapping generations. Econometrica, 53(6):1499-1528. 
Yi, S., Xu, Z., and Wang, G.-J. (2018). Volatility connectedness in the cryptocurrency market: Is Bitcoin a dominant cryptocurrency? International Review of Financial Analysis. 